\title{
Compound Controller for DC Motor Servo System Based on Inner-Loop Extended State Observer
}

\author{
Jingxian Yang \\ Electrical Engineering College of Northwest University for Nationalitie, Lanzhou 730100, China \\ Email: yangjxian12@126.com
}

\begin{abstract}
As DC motor servo systems are more and more widely applied in the manufacturing industry and aerospace domain, the requirements on control performance are increased by the complicated various working environments. With regard to the uncertainties including modeling error, parameter variations and external disturbances in DC motor servo system, one Nonlinear Extended Disturbance Observer (NESO) is constructed, and its output will be used as the design reference of disturbance compensation term in control system. Based on the as-built NESO in inner loop, one outer-loop compound controller by means of state-space design method is proposed in order to realize the high-precision position tracking ability of the servo system. Computer simulation results show that compared with conventional control schemes, the proposed control scheme can guarantee fewer tracking errors of DC motor servo system. Moreover, it possesses stronger robustness against system uncertainties including modeling error, parameter variations and friction moment disturbance.
\end{abstract}

Keywords: Motor servo system, Extended State Observer (ESO), Active Disturbance Rejection Control (ADRC), compound control, robust control.

\section{Introduction}

With the development of modern magnetic material - the rare earth alloy, which has been successfully used in brushless DC motor, the motor response speed, torque and power/mass ratio have been greatly improved [1]. Therefore, DC motor is widely used in many kinds of high-precision position servo systems, including opto-electronic tracking system [2], missile electro-mechanical actuator [3] and electric flight simulator [4] and so on. At the same time, the performance requirements on DC motor servo system are becoming higher and higher. However, due to the existence of uncertainties, including modeling errors, parameter perturbations and disturbance torques, the performance enhancement of motor servo 
system will be limited greatly $[5,6]$. As a result, it is necessary to explore some new and practical control technologies for servo motor.

Active Disturbance Rejection Control (ADRC) is such a technology that it can estimate the current system uncertainties in real time and then compensate the same amount of control quantity. This control technology does not need accurate model information and it either does not need to assume that the model is linear or bound, therefore it can deal with a large range of uncertain systems [8-10]. In fact, the strong robust property of ADRC owes to the introduction of Extended State Observer (ESO), which can estimate the current uncertain dynamics of system more accurately and then make compensations to the control system. Moreover, the control system, after being compensated, has the advantages of simple structure and easy implementation. In this paper, a Nonlinear Extended State Observer (NESO) is constructed to be applied in inner-loop control of DC motor servo system, in order to ensure the robust performance of control system.

In automatic control systems, if a feedforward path is added to the feedback control loop, a compound control system will be constructed. When the system parameters are chosen properly (usually according to the invariance principle), the control system is not only stable, but it also greatly reduces or even eliminates the steady state error. Therefore, when the compound control technology is applied in the position servo system, it can realize the high-speed and high-precision tracking for position command [11, 12]. To sum it up, the servo control scheme, which is composed of ESO and compound controller, can not only suppress the influences of external disturbances and parameters perturbation, but also improve the position tracking accuracy of servo system, which is the main starting point of this paper.

The remainder of this paper is organized as follows. In Section 2, the mathematical modelling on DC motor servo system is carried out. In Section 3, according to the as-built model, the inner-loop NESO and the outer-loop compound controller are respectively designed based on the dual-loop control thought. Compared with the traditional control schemes, the performance advantages of the proposed control scheme are verified by computer simulation in Section 4. In addition, the corresponding conclusions are shown in Section 5.

\section{Problem description}

A typical DC motor servo control system is composed of servo controller, power amplifier, DC motor, loads, measuring elements and so on, and its structure is shown in Fig. 1.

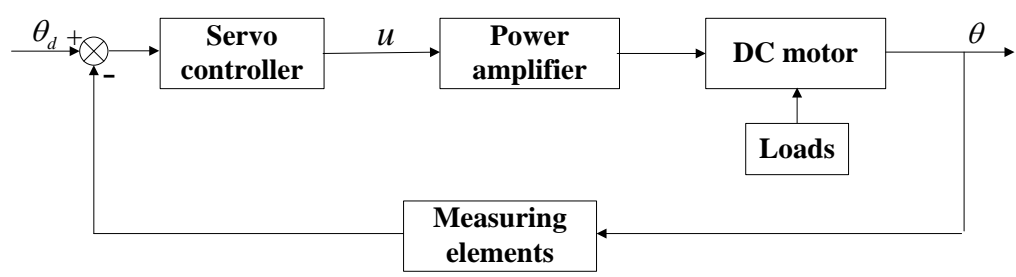

Fig. 1. Structural diagram of DC motor servo system 
where $\theta_{d}, u$ and $\theta$ denote the angle position command, servo controller output and actual output angle of DC motor servo system, respectively.

Considering the existence of equivalent disturbance factors (including modeling errors, parameters perturbation and external disturbances), and assuming that the connection between the motor and the loads is rigid, the DC motor servo system can be described as follows [2,3]:

$$
J \ddot{\theta}(t)+B \theta(t)=u(t)+d(t),
$$

where $J>0$ and $B>0$ respectively denoting the equivalent moment of inertia and the equivalent damping coefficient of the whole DC motor servo system; $d$ denotes the equivalent disturbances at the input of servo system, and it is assumed to be bounded, i.e., $|d| \leq M$.

Let $x_{1}=\theta(t)$ and $x_{2}=\dot{\theta}(t)$ respectively denote the actual angle and the angular speed of DC motor servo system. Then, the original Equation (1) can be re-written in the following state-space expression:

$$
\left\{\begin{array}{l}
\dot{x}_{1}=x_{2}, \\
\dot{x}_{2}=-\frac{B}{J} x_{2}+\frac{1}{J} u(t)+\frac{1}{J} d(t) .
\end{array}\right.
$$

Until now, the mathematical model of DC motor servo system has been established, and it lays the basic foundation for the next design of servo controller, which will be presented in next section.

\section{Servo controller design}

\subsection{Inner-loop NESO design}

If the equivalent disturbances $d$ in system (2) can be estimated by means of some observation methods, and then the same amount of compensation is introduced into control input, the influences of equivalent disturbances on control system performance will be effectively suppressed. Based on this control thought, a kind of NESO, which is used as the inner-loop controller of servo motor system, is constructed according to servo plant model (2).

In practical engineering, the parameters $(J$ and $B)$ of servo system can be obtained by means of some measurement or estimation methods, and their nominal values will be used in the design process. Based on this, Equation (2) can be rewritten as

$$
\left\{\begin{array}{l}
\dot{x}_{1}=x_{2}, \\
\dot{x}_{2}=-\frac{B_{n}}{J_{n}} x_{2}+\frac{1}{J_{n}} u(t)+\bar{d}(t),
\end{array}\right.
$$

where $\bar{d}(t)$ denotes system uncertainties, which include modelling errors, parameters perturbation and external disturbances, and its expression is shown as follows: 


$$
\bar{d}(t)=\left(\frac{1}{J}-\frac{1}{J_{n}}\right) u(t)+\left(\frac{B_{n}}{J_{n}}-\frac{B}{J}\right) x_{2}+\frac{1}{J} d(t) .
$$

Therefore, $\bar{d}(t)$ can be regarded as the state variable to be extended, meanwhile Equation (4) provides the theoretical basis for verifying the accuracy of disturbance estimation in further simulation.

Aiming at the uncertain system (3), we put forward a construction method of NESO, which is shown as follows:

$$
\left\{\begin{array}{l}
e=z_{1}-x_{1}, \\
\dot{z}_{1}=z_{2}-\beta_{0} e \\
\dot{z}_{2}=z_{3}-\frac{B_{n}}{J_{n}} z_{2}+\frac{1}{J_{n}} u(t)-\beta_{1} \operatorname{fal}(e, a / 2, \delta), \\
\dot{z}_{3}=-\beta_{2} \operatorname{fal}(e, a / 4, \delta),
\end{array}\right.
$$

where $z_{1}$ and $z_{2}$ are the estimation variables with respect to state variables $x_{1}$ and $x_{2}$, respectively; $z_{3}$ is the estimation variable with respect to uncertain variable $\bar{d}$; the other parameters are $\beta_{0}>0, \beta_{1}>0, \beta_{3}>0, a>0, \delta>0$. In addition, the nonlinear function is defined as follows:

$$
\operatorname{fal}(e, \alpha, \delta)=\left\{\begin{array}{ccc}
|e|^{\alpha} \operatorname{sgn}(e) & \text { if } & |e|>\delta, \\
\frac{e}{\delta} & \text { if } & |e| \leq \delta .
\end{array}\right.
$$

Therefore, the state $z_{3}$ of NESO will be used as the design basis of disturbances compensation in the servo control system. Furthermore, the term of disturbances compensation can be designed as

$$
\hat{d}(t)=J_{n} z_{3} .
$$

Then, we can get the corresponding inner-loop control law $-\hat{d}(t)$. Moreover, the inner-loop control structure based on NESO is shown in Fig. 2, where $v$ denotes the output of outer-loop controller.

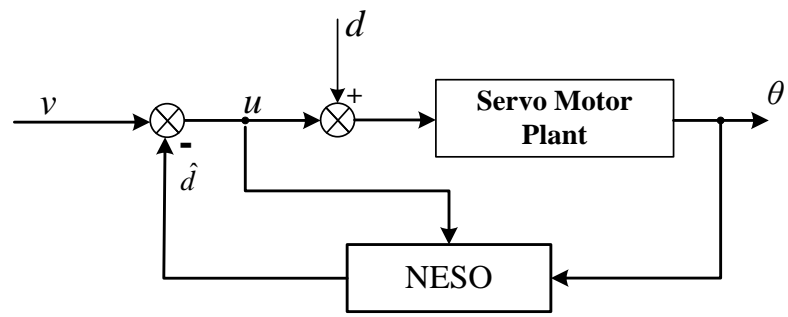

Fig. 2. Structural diagram of inner loop control based on NESO

Note that, the as-built NESO in this subsection can only realize the robust performance of the inner-loop control system, and it cannot guarantee the position tracking function of DC motor servo system. Therefore, an outer-loop controller based on the adjustment of position errors is also needed, and its design process will be presented in next subsection. 


\subsection{Outer-loop compound controller design}

According to the previous analysis, the system (2) can be rewritten as the following form after introducing the disturbances compensation from NESO:

$$
\left\{\begin{array}{l}
\dot{x}_{1}=x_{2}, \\
\dot{x}_{2}=-\frac{B_{n}}{J_{n}} x_{2}+\frac{1}{J_{n}}[v(t)-\hat{d}(t)]+\bar{d}(t) \approx-\frac{B_{n}}{J_{n}} x_{2}+\frac{1}{J_{n}} v(t) .
\end{array}\right.
$$

For the servo motor plant, we can design the outer-loop controller by using compound structure, that is, the combination of PD feedback control and feedforward control, which is shown in the following diagram.

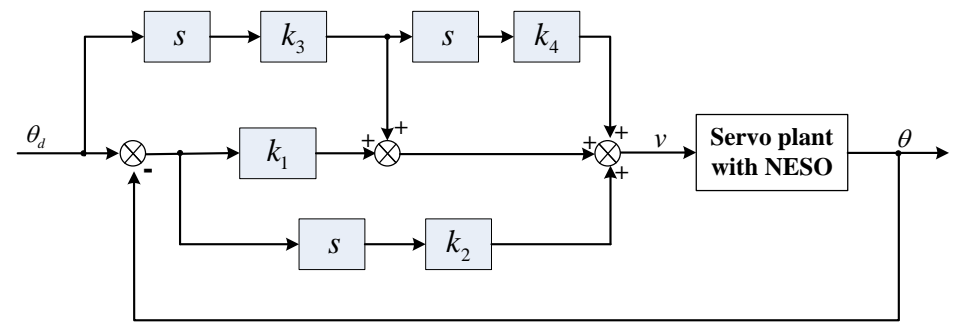

Fig. 3. NESO structural diagram of compound control

In next, the selection principle of the four control coefficients in Fig. 3 will be obtained by using the state-space design method.

In the first, we define the state variable $\mathbf{x}=\left[\begin{array}{ll}x_{1} & x_{2}\end{array}\right]^{\mathrm{T}}$, and then rewritten Equation (8) into the general formation of state space, which is shown as follows:

$$
\dot{\mathbf{x}}=\mathbf{A}_{n} \mathbf{x}+\mathbf{b}_{n} v(t),
$$

where

$$
\mathbf{A}_{n}=\left[\begin{array}{cc}
0 & 1 \\
0 & -\frac{B_{n}}{J_{n}}
\end{array}\right], \quad \mathbf{b}_{n}=\left[\begin{array}{c}
0 \\
1 \\
J_{n}
\end{array}\right] .
$$

Then, make the variable substitution as follows:

$$
\mathbf{x}_{e}=\mathbf{x}_{d}-\mathbf{x}, \quad \mathbf{x}_{d}=\left[\begin{array}{ll}
\theta_{d} & \dot{\theta}_{d}
\end{array}\right]^{\mathrm{T}},
$$

where $\dot{\theta}_{d}$ denotes the angular speed command of DC motor servo system. As a result, the equivalent system corresponding to (9) can be described as

$$
\dot{\mathbf{x}}_{e}=\mathbf{A}_{n} \mathbf{x}_{e}+\mathbf{b}_{n} v_{1}(t),
$$

where

$$
v_{1}(t)=-v(t)+J_{n} \ddot{\theta}_{d}+B_{n} \dot{\theta}_{d} .
$$

Moreover, $\ddot{\theta}_{d}$ denotes the angular acceleration command of DC motor servo system.

Therefore, the tracking problem of the original system (9) will evolve into the regulation problem of the equivalent system (11), which can greatly simplify the design process [3]. Aiming at the equivalent system (11), we can design the feedback control law as follows: 


$$
v_{1}(t)=-\mathbf{k} \mathbf{x}_{e}=-\left[\begin{array}{ll}
k_{1} & k_{2}
\end{array}\right] \mathbf{x}_{e} .
$$

According to the theory of pole assignment, the selection principle of the feedback controller coefficients can be obtained. That is, by choosing the appropriate state feedback gain vector $\mathbf{k}$, we make all the eigenvalues of matrix $\mathbf{A}_{n}-\mathbf{b}_{n} \mathbf{k}$ possess negative real parts. In addition, because the equivalent system $\left(\mathbf{A}_{n}, \mathbf{b}_{n}\right)$ is controllable, we can assign the locations of its eigenvalues to any desired positions.

According to Equations (12)-(13), the output of outer-loop controller can be described as

$$
v(t)=\mathbf{k} \mathbf{x}_{e}+J_{n} \ddot{\theta}_{d}+B_{n} \dot{\theta}_{d} .
$$

According to the block diagram of compound control system, the basis for coefficients selection of the feedforward controller can be obtained as:

$$
k_{3}=B_{n}, k_{4}=\frac{J_{n}}{B_{n}} .
$$

In summary, for the DC motor servo system (2), the control law consists of two parts, which can be expressed as follows according to Equations (7) and (14):

$$
u(t)=v(t)-\hat{d}(t)=\mathbf{k} \mathbf{x}_{e}+J_{n} \ddot{\theta}_{d}+B_{n} \dot{\theta}_{d}-J_{n} z_{3} .
$$

Until now, the robust controller has been completed for DC motor servo system, and the next sequel will carry out computer simulation in order to verify its effectiveness.

\section{Simulation results}

In this section, we will compare the control effects of the proposed scheme with the traditional PD scheme and PD+NESO scheme by using computer simulation, and then the performance advantages of the proposed control scheme can be obtained.

In simulation, the actual plant parameters of DC motor servo system are shown as

$$
J=0.0022 \mathrm{~N} . \mathrm{s}^{2} \text { per } 1^{\circ}, B=0.55 \mathrm{~N} . \mathrm{s}^{2} \text { per } 1^{\circ} .
$$

In consideration of the fact that there must exist some modeling errors in practical engineering, the values of nominal parameter are chosen as:

$$
J_{n}=0.002 \mathrm{~N} . \mathrm{s}^{2} \text { per } 1^{\circ}, B_{n}=0.05 \mathrm{~N} . \mathrm{s}^{2} \text { per } 1^{\circ} \text {. }
$$

In addition, the influences of Coulomb friction torque and plant parameters perturbation are added in DC motor servo control system, meanwhile the equivalent disturbances caused by the above two factors are $-3.0 \operatorname{sgn}(\dot{\theta})$ and $1.0 \cos (2 \pi t)$, respectively. Assuming that the initial angle position of motor servo system is set as $0.2^{\circ}$, the initial angular velocity is set as zero. At the same time, the maximum value of servo controller output is $10 \mathrm{~V}$.

The corresponding coefficients of PD controller are:

$$
k_{1}=3.0, k_{2}=0.25 \text {. }
$$

The corresponding coefficients of feedforword controller are:

$$
k_{3}=0.05, \quad k_{4}=0.04 \text {. }
$$


The corresponding coefficients of NESO are:

$$
\beta_{0}=1000, \quad \beta_{1}=10000, \quad \beta_{3}=500000, \quad a=1, \delta=0.01 \text {. }
$$

In the simulation, a composite signal is selected as the position tracking command of motor servo system, as shown in Fig. 4.

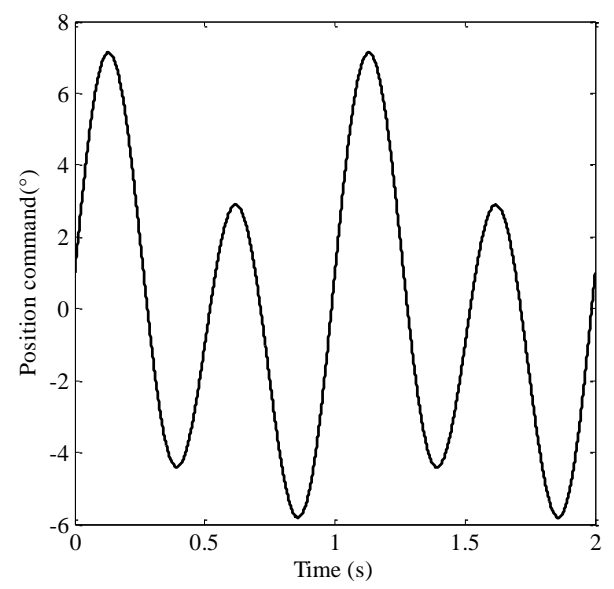

Fig. 4. Curve of position tracking command

Taking the composite signal as the input command, the position tracking errors of motor servo systems under the three control schemes are compared in the following, as shown in Fig. 5.

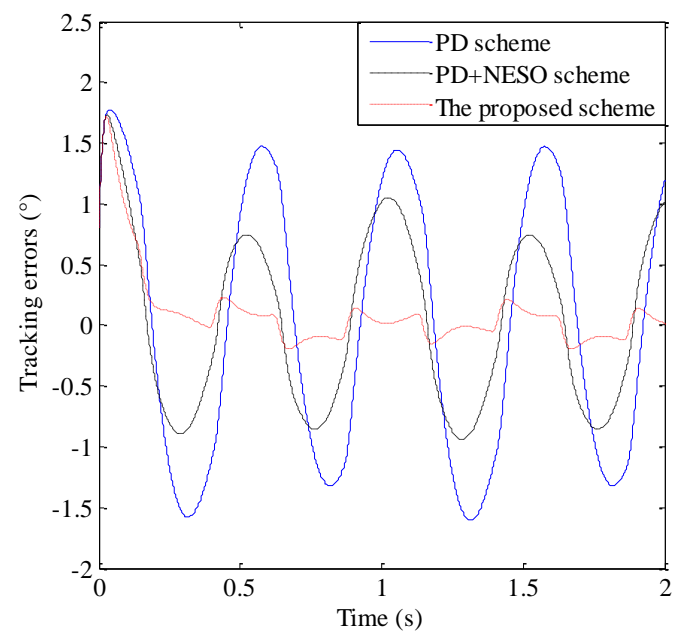

Fig. 5. Curves of position tracking errors of motor servo systems under the three control schemes

As can be seen from Fig. 5, when NESO is introduced into the traditional PD control scheme, that is the PD+NESO control scheme is formed, the tracking errors of motor servo system can be significantly reduced compared with traditional PD control scheme. This reflects the robust property of as-built NESO against system uncertain dynamics. Furthermore, when the compound controller is introduced, that 
is the proposed control scheme is formed, the tracking errors of motor servo system will be greatly reduced, which reflects its effectiveness to improve the tracking accuracy of servo motor.

In fact, the robustness of NESO comes from its real-time and accurate estimation on system uncertain dynamics. In order to verify this point, the state variable $z_{3}$ of NESO used to estimate the extended variable (Fig. 6).

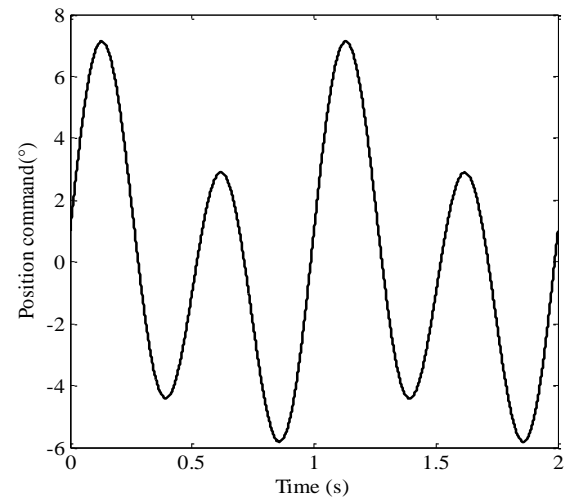

Fig. 6. Estimation curve about extended variable by using NESO

As can be seen from Fig. 6, the state variable $z_{3}$ of NESO can estimate the extended variable $\bar{d}$ real-timely and accurately. Because the extended variable is the real reflection of the system dynamics, the inner-loop controller based on NESO can effectively compensate the effect of uncertain dynamics on system performance, which further ensures the validity of the proposed control scheme. At the same time, the control quantity of motor servo system under the proposed control scheme is presented in Fig. 7.

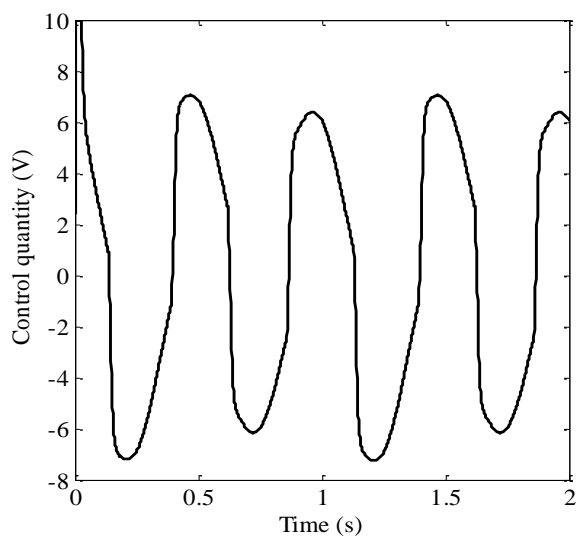

Fig. 7. Curve of control quantity of motor servo system

As seen in Fig. 7, the control quantities of DC motor servo system are relatively smooth, which avoids the influences of high-frequency chattering on system stability and the damage to the mechanical structure. Therefore, it enhances the practical value of the control scheme proposed in this paper. 


\section{Conclusions}

In view of DC motor servo system, this paper presents a double loop servo controller, which is based on the combination of an inner-loop NESO and an outerloop compound controller, and it can ensure the stable, fast and accurate position tracking performance of DC motor. At the same time, the designed NESO can deal with a large range of uncertain dynamics in control system, including modelling errors, parameters perturbation, external disturbances and so on, which can ensure the proposed control scheme possess strong robustness. Simulation results show that, the proposed control scheme has a better position tracking performance compared with the traditional control schemes. In addition, its structure is simple and easy to be implemented in practice, and the experimental verification will be given in future works.

\section{References}

1. Li, F. X., W. J. Zhu. Research and Application of Dual-Mode Operation Technology of Brushless DC Motor. - [J], Vol. 17, 2013, No 3, pp. $70-75$ (in Chinese).

2. Re n, Y., Z. H. Li u, R. Z hou. Application of Low Speed Opto-Electronic Tracking Systems Based on Sliding Mode Distutbance Observer. - Journal of Beijing University of Aeronautics and Astronautics, Vol. 39, 2013, No 6, pp. 835-840 (in Chinese).

3. Liu, X. D., Y. J. Wu, Y. Deng et al. A Global Sliding Mode Controller for Missile Electromechanical Actuator Servo System. - Proc. IMechE. Part G: Journal of Aerospace Engineering, Vol. 228, 2014, No 7, pp. 1095-1104.

4. Niu, J. J., Y. L. Fu, S. H. Liu. Inner Axis Control with Friction Compensation for High Precision Flight Motion Simulator. - Vol. 12, 2008, No 5, pp. 576-579 (in Chinese).

5. Ch o i, H. S., Y. H. P a r k, Y. S. C h o et al. Global Sliding Mode Control Improved Design for a Brushless DC Motor. - IEEE Control Systems Magazine, Vol. 21, 2001, No 3, pp. 27-35.

6. K i m, B. K., W. K. Chung, K. O h b a. Design and Performance Tuning of Sliding-Mode Controller for High-Speed and High-Accuracy Positioning Systems in Disturbance Observer Framework. - IEEE Transactions on Industrial Electronics, Vol. 56, 2009, No 10, pp. 3798-3809.

7. H a n, J. Q. Active Disturbance Rejection Control Technique-the Technique for Estimating and Compensating the Uncertainties. Beijing, National Defense Industry Press, 2007 (in Chinese).

8. X i a, Y., P. S h i, G. L i u. Active Disturbance Active Control for Uncertain Multivariable Systems with Time-Delay. - IET Control Theory and Application, Vol. 1, 2007, No 1, pp. 75-81.

9. G u o, B. Z., F. F. J i n. Sliding Mode and Active Disturbance Rejection Control to Stabilization of One-Dimensional Anti-Stable Wave Equations Subject to Disturbance in Boundary Input. IEEE Transactions on Automatic Control, Vol. 58, 2013, No 5, pp. 1269-1274.

10. G u o, B. Z., F. F. J i n. The Active Disturbance Rejection and Sliding Mode Control Approach to the Stabilization of Euler Euler-Bernoulli Beam Equation with Boundary Input Disturbance. - Automatica, Vol. 49, 2013, No 9, pp. 2911-2918.

11. Li u, H., W. G a o. Study of the Feedforward Friction Compensation in Servo System. - Science Technology and Engineering, 2007, No 4, pp. 614-616 (in Chinese).

12. D i n g, Q. Q. Technical Study of Compound Control about the Radar of Ship-Borne. - Science Technology and Engineering, Vol. 13, 2013, No 15, pp. 4197-4200 (in Chinese). 